\title{
VALIDAÇÃO DE INSTRUMENTO DE COLETA DE DADOS DE ENFERMAGEM EM UMA UNIDADE DE TERAPIA INTENSIVA PEDIÁTRICA
}

\section{VALIDATION OF NURSING DATA COLLECTION INSTRUMENT IN A PEDIATRIC INTENSIVE CARE UNIT}

\author{
Clara Santana Sousa ${ }^{1} *$ Jessika Valeska Martins $\operatorname{Ramos}^{2} *$ Joseilze Santos de Andrade $^{3} *$ \\ Yara Mercedes Oliveira Santos ${ }^{4}$ Thialla Andrade Carvalho 5
}

\begin{abstract}
RESUMO
Objetivo: Validar um instrumento de coleta de dados de enfermagem em Unidade de Terapia Intensiva Pediátrica. Metodologia: Estudo metodológico de validação, desenvolvido em três fases: construção de instrumento de coleta de dados de enfermagem, testagem e validação. O processo de validação foi realizado por quatro enfermeiros pesquisadores e cinco enfermeiros assistenciais do setor estudado no período de janeiro e fevereiro de 2018. A análise foi realizada pelos cálculos do Índice de Validação de Conteúdo, coeficiente de Kendall e Kappa de Cohen, sendo considerados validados os itens que atingiram Índice $\geq 70 \%$ e significância $<0,05$ para os últimos dois cálculos. Resultados:93\% dos itens avaliados pelos enfermeiros atingiram Índice de Validação de Conteúdo $\geq 70 \%$, enquanto os experts validaram $57 \%$ dos itens. Entretanto, os outros cálculos apresentaram índice de significância maior que o estabelecido, demonstrando não concordância entre os validadores. Após a reformulação do instrumento com as sugestões da primeira rodada, houve apenas considerações do expert 2 , que foram analisadas e alteradas no instrumento, alcançando um IVC de 100\%. Conclusão: Alcançou-se a validação de todo o instrumento, facilitando a implementação da primeira etapa do processo de enfermagem. Implicações para prática: disponibilizar um instrumento validado que contribua para prática da assistência de enfermagem.
\end{abstract}

Palavras-chave: Processo de Enfermagem; Estudos de Validação; Cuidados Intensivos; Pediatria; Segurança do Paciente.

\section{ABSTRACT}

Objective: Validate a Nursing data collection instrument in a pediatric intensive care unit. Method: Methodological study of validation developed in three phases: elaboration of a Nursing data collection instrument, test process and validation. The validation process had been carried out by four researchers' nurses and five treating nurses of the evaluated sector during the period of January and February. The analysis has been performed by the calculations of Content Validation Index, Kendall e Kappa de Cohen Coefficient, among the results was considered validated those items that achieved index $\geq 70 \%$ and significance $<0,05$ to the last two calculations. Results: $93 \%$ of the items evaluated by the nurses reached Content Validation Index $\geq 70 \%$ whereas the experts validated $57 \%$ of the items. Meanwhile, the others calculations presented greater significance index than the established one, indicating non-concordance between the validators. After instrument's reformulation with the suggestions of the first round, there was only comments of the expert number 2, which were analysed and changed in the second round instrument, thus achieving an IVC of $100 \%$. Conclusion: Validation of the entire instrument was achieved, facilitating the deployment of the first phase of nursing process.

Keywords: Nursing Process; Validation Study; Intensives Care; Pediatric; Patient Safety.

\footnotetext{
${ }^{1}$ Universidade Federal de Sergipe (UFS). E-mail: clarasantanasousa@ gmail.com

${ }^{2}$ Orcid: https://orcid.org/0000-0001-7449-3694 E-mail: jessikaramosenfa@hotmail.com

${ }^{3}$ Orcid: https://orcid.org/0000-0003-0488-2840 E-mail: joseilzesa@ gmail.com

${ }^{4}$ Orcid: https://orcid.org/0000-0001-9425-3591 E-mail: yarapituca34@ gmail.com

${ }^{5}$ Orcid: https://orcid.org/0000-0002-3301-5060 E-mail: thiallaba@hotmail.com
} 


\section{INTRODUÇÃO}

As Unidades de Terapia Intensiva Pediátrica (UTI-Ped) atendem crianças com casos clínicos e cirúrgicos graves por meio da utilização de tecnologias avançadas, cuidados especializados e procedimentos complexos e invasivos. Neste cenário, a tomada de decisão adequada é fator crucial para salvar vidas, sendo necessário habilidades e conhecimento científico apurados para o desenvolvimento de atividades complexas, respaldadas no saber científico. $^{1}$

$\mathrm{O}$ Estatuto da Criança e do Adolescente (ECA) em seu artigo $2^{\circ}$, dispõe que a infância contempla indivíduos até os doze anos de idade incompletos. ${ }^{2}$ Dessa forma, é necessário entender as especificidades de cada faixa etária para atender as reais necessidades apresentadas por esses indivíduos, pois a ausência dessas informações pode gerar complicações para criança, família e profissionais da saúde envolvidos no processo de cuidado. ${ }^{3}$

Devido às particularidades dessa clientela e do nível de complexidade em saúde que exigem quando em regime de tratamento intensivo, torna-se necessário que os profissionais de enfermagem utilizem metodologias de organização do serviço e apliquem instrumentos que facilitem a comunicação e o raciocínio diagnóstico, contribuindo para melhoria da qualidade da assistência, segurança do paciente, promovendo uma assistência individualizada e cientifica, para isso $\mathrm{o}$ processo de enfermagem torna-se essencial. ${ }^{4-6}$

Alguns dos desencadeamentos de eventos adversos na prática de enfermagem estão principalmente relacionados aos cuidados de rotina. ${ }^{6}$ Dessa forma, as crianças que já são susceptíveis a infecções e lesões devido as suas peculiaridades anatômicas, fisiológicas e imunológicas ficam vulneráveis a negligência de cuidados podendo agravar ainda mais o seu estado de saúde.?

$\mathrm{O}$ cuidar para enfermagem precisa ser integral, sistematizado e de máxima eficiência. ${ }^{8}$ Com o intuito de subsidiar a Sistematização da Assistência de Enfermagem (SAE) e a implementação do Processo de Enfermagem (PE) em ambientes públicos ou privados, em que ocorre o cuidado profissional de Enfermagem, que a Resolução do Conselho Federal de Enfermagem COFEN 358/2009, em seu Art. 2º delibera que o processo de enfermagem se organiza em cinco etapas inter-relacionadas, interdependentes e recorrentes, sendo a primeira delas a Coleta de dados de Enfermagem. ${ }^{9}$

Nesse raciocínio, este estudo justificase pela necessidade de disponibilizar um instrumento de coleta de dados padronizado e validado que direcione o olhar e a prática do enfermeiro na busca pelo atendimento das necessidades humanas básicas e, com isso, evite/reduza a ocorrência de agravos à saúde 
das crianças assistidas, além de cumprir a legislação específica da área.

Para que o processo de enfermagem não se fragilize é imprescindível que se escolha um referencial teórico que garanta a concordância entre filosofia, necessidades da clientela e teoria e estruture o instrumento a ser criado. ${ }^{10}$ Considerando que a Teoria das Necessidades Humanas Básicas(NHB) de Wanda Horta representa uma das teorias mais utilizadas em terapia intensiva e atende as necessidades da clientela do estudo em questão, esta, foi escolhida para subsidiar a construção do instrumento de coleta de dados. $^{3}$

Diante do exposto, surgiu o questionamento: como se apresenta um instrumento de coleta de dados para aplicação do processo de enfermagem em uma Unidade de Terapia Intensiva Pediátrica (UTI-Ped) após os processos de construção e validação?

Assim, este estudo teve como objetivo validar um instrumento de coleta de dados de enfermagem em uma Unidade de Terapia Intensiva Pediátrica.

Por meio da busca em bases de dados sobre diagnósticos de enfermagem prevalentes em UTI-Ped, foi verificada uma lacuna de abordagem desse perfil diagnóstico para o público pediátrico. Este achado indicou a relevância para a elaboração deste trabalho, a fim de colaborar com a produção do conhecimento sobre o assunto.

\section{MÉTODO}

Estudo metodológico de validação, desenvolvido em três fases: construção do instrumento de coleta de dados de enfermagem, testagem e validação de conteúdo e aparência. Realizado em Unidade de Terapia Intensiva Pediátrica de um hospital terciário de grande porte, público do Nordeste do Brasil cujo público alvo constou de crianças com idade entre 28 dias e 11 anos, 11 meses e 29 dias em cuidados intensivos, entre novembro de 2018 e setembro de 2019. Participaram da pesquisa enfermeiros assistenciais (fases de testagem e validação) e pesquisadores (fase de validação).

Estudo aprovado pelo Comitê de Ética e Pesquisa com Seres Humanos da Universidade Federal de Sergipe, sob CAAE $\mathrm{n}^{\mathrm{o}}$ 65780616.2.0000.5546, pelo parecer consubstanciado $\mathrm{n}^{\mathrm{o}} 2.203 .882$, respeitando os aspectos éticos e legais.

Inicialmente foi realizado um levantamento de literatura sobre as necessidades humanas básicas apontadas por Wanda Horta ${ }^{11}$ e sobre a Taxonomia NANDAI. ${ }^{12}$

A construção do instrumento ocorreu em dezembro de 2018, considerando a teoria e o sistema de classificação de diagnósticos de enfermagem escolhidos, de modo a direcionar a construção de diagnósticos de enfermagem. Além de itens inerentes à assistência em terapia intensiva pediátrica, aspectos semiológicos e tecnológicos, dada a 
existência de procedimentos complexos e invasivos nesse cenário de cuidado.

Para verificar a aplicabilidade do instrumento, realizou-se uma testagem prática por enfermeiros assistenciais em, pelo menos, duas crianças internadas no setor estudado. Após a testagem, os enfermeiros preencheram o roteiro de avaliação elaborado para esse fim. Foram incluídos todos os enfermeiros alocados no setor durante $\mathrm{o}$ período de coleta de dados, que aceitaram contribuir com a pesquisa assinando o Termo de Consentimento Livre e Esclarecido (TCLE). Esta fase ocorreu entre fevereiro e março de 2019.

Em seguida realizou-se a validação de conteúdo e aparência por enfermeiros pesquisadores (Experts) e assistenciais. Os primeiros foram selecionados pela técnica de Delphi, um método sistematizado de avaliar informações, obter consenso de especialistas sobre determinado tema por meio de validações organizadas em fases. É realizado de forma coletiva e aplicada a dados quantitativos ou qualitativos. ${ }^{13}$

Os critérios para seleção foram divididos em dois grupos: critérios indispensáveis e critérios individuais. $\mathrm{O}$ primeiro grupo englobou os critérios: ser enfermeiro (a) e possuir doutorado; e o segundo grupo: experiência com processo de enfermagem, e/ou pediatria, e/ou em unidades de terapia intensiva, e/ou em estudos de validação.
Primeiramente, houve a seleção dos experts na Plataforma Lattes, que gerou uma amostra inicial de 24 pesquisadoras. A seguir, foi enviado um e-mail contendo a Carta Convite para participação e o TCLE. Em caso de aceite, uma cópia do TCLE assinado foi enviada às pesquisadoras confirmando a participação. Dos 24 e-mails enviados, três endereços eletrônicos foram bloqueados pelo provedor; duas pesquisadoras responderam rejeitando o convite; e seis responderam aceitando participar, sendo que apenas quatro enviaram o instrumento de avaliação respondido, o que configurou a amostra final de quatro validadoras.

Com a finalidade de contribuir no processo de validação e, tendo em vista a experiência clínica comprovada, os enfermeiros assistenciais que realizaram a testagem do instrumento na segunda fase da pesquisa, foram convidados para participar, também, como validadores do referido instrumento.

Para aqueles que aceitaram participar, o instrumento de coleta de dados a ser validado e o roteiro de avaliação foram enviados/entregues às especialistas, com prazo de 15 dias para devolução. Esta etapa transcorreu entre abril e maio de 2019.

$\mathrm{O}$ roteiro de avaliação foi organizado em 14 dimensões, que dizem respeito às necessidades avaliadas, sendo que cada uma delas contempla cinco itens: adequação do 
modelo proposto, conteúdo, aparência, clareza/compreensão, objetividade.

Para avaliação dos itens de cada dimensão, foram utilizados graus variados de concordância da escala Likert de três pontos, a saber: 1 (inadequado); 2 (necessita de reformulação); 3 (adequado). ${ }^{14}$ Os itens avaliados com pontuação um ou dois, foram acompanhados de justificativas e sugestões pelos avaliadores.

Após resultados da avaliação da primeira rodada, os dados foram tabulados no Microsoft Excel versão 2013 e analisados estatisticamente para determinar a fidedignidade e a validade da avaliação.

Para consolidar a validação de conteúdo, foi utilizado o Índice de Validação de Conteúdo (IVC), no qual para o item ser considerado validado, deveria atingir um índice $\geq 70 \%$. Foi realizada uma avaliação conjunta sobre todos os itens utilizando o coeficiente de concordância de Kendall. ${ }^{15}$ Para verificação da concordância aos pares, utilizou-se a estatística Kappa de Cohen. ${ }^{16}$ Ambas as análises estatísticas possuem variação entre 0 e 1 , em que, quanto mais próximo de 1 , maior será a concordância entre os juízes. Em todos os casos a significância da estatística foi feito um teste de hipótese, cujo resultado é dado através do p-valor, cuja concordância entre os juízes foi considerada significativa quando p-valor < 0,05 . Todas as análises foram realizadas no software R, versão 3.4.0.

\section{RESULTADOS}

A faixa etária média dos validadores foi de 42,4 anos, sendo sete $(77,77 \%)$ do sexo feminino, sete $(77,7 \%)$ residentes na Região Nordeste e dois $(22,2 \%)$ da Região Sudeste. Todos, nove (100\%), graduaram-se em instituições universitárias públicas e tiveram atuação na assistência, alguns ainda estão envolvidos com a docência, sendo quatro $(44,4 \%)$ doutores.

$\underline{\text { Análise quantitativa dos dados }}$

O cálculo do IVC foi realizado pela razão entre o número de respostas "aprovado (3)" e o número de respostas que cada item obteve. Foram considerados validados os que atingiram IVC $\geq 70 \%$. Aproximadamente $57 \%$ (40) e 93\% (65) dos itens avaliados pelos experts e assistenciais, respectivamente, atingiram IVC significante na primeira etapa.

Alguns itens não foram avaliados segundo a escala Likert, o que interferiu no IVC positiva ou negativamente. Na avaliação dos enfermeiros, apenas cinco itens não obtiveram IVC $\geq 70 \%$. Vale destacar que em todos os itens em que não foi atingida significância pela avaliação dos enfermeiros, também não houve significância pela avaliação dos experts, o que pode representar que houve concordância de avaliação entre eles.

Na tabela 1, observa-se que nenhum $\mathrm{p}$ valor foi menor que o nível de significância adotado $(0,05)$. Os experts não apresentaram 
concordância entre eles, tanto no total, quanto

em algum domínio.

Tabela 1- Concordância conjunta dos Experts, avaliado através do coeficiente de concordância de Kendall. Aracaju, SE, Brasil, 2018.

\section{Dimensão}

\section{Concord. De Kendall}

0.16

0.11

0.7816

0.11

0.7816

0.10

0.7530

0.10

0.7530

0.13

0.7358

0.20

0.5169

0.20

0.5169

0.10

0.7530

0.11

0.7816

0.14

0.5698

0.28

0.3425

0.20

0.5169

0.00

1.0000
Na tabela 2 foram avaliados todos os pares de experts em relação a sua concordância. Apenas em um caso foi observada concordância significativa, (P-valor $=0,008)$. 
Tabela 2 - Concordância aos pares dos Experts, através do coeficiente de concordância de Kappa de Cohen. Aracaju, SE, Brasil, 2018.

$\begin{array}{llll}\text { Expert } 1 & \text { Expert } 2 & \text { Expert } 3 & \text { Expert } 4\end{array}$

Expert 1

Expert $2 \quad 0.00(1.000)$

$\begin{array}{lll}\text { Expert } 3 & 0.00(1.000) & 0.07(0.455)\end{array}$

$\begin{array}{lll}\text { Expert } 4 & 0.00(1.000) & 0.20(0.093)\end{array}$

$0.264(0.008)$

Fonte: Os autores

Em relação aos enfermeiros, também resultado foi ocasionado pelo julgamento do não foi observada concordância conjunta, Enfermeiro 1, que discordou dos demais. conforme observado na tabela 3. Esse

Tabela 3 - Concordância conjunta dos Enfermeiros, através do coeficiente de concordância de Kendall. Aracaju, SE, Brasil, 2018.

\begin{tabular}{ccc} 
Dimensão & Concord. De Kendall & P-valor \\
\hline 1 & 0.03 & 0.963 \\
2 & 0.00 & 1.000 \\
3 & 0.03 & 0.963 \\
4 & 0.03 & 0.963 \\
5 & 0.02 & 0.983 \\
6 & 0.00 & 1.000 \\
7 & 0.07 & 0.844 \\
8 & 0.03 & 0.963 \\
9 & 0.00 & 1.000 \\
10 & 0.00 & 1.000
\end{tabular}


Fonte: Os autores

Na tabela 4, que representa a avaliação aos pares, nota-se que houve uma concordância entre os Enfermeiros 2, 3, 4 e 5, porém o Enfermeiro 1 não apresentou concordância com nenhum dos outros avaliadores. Chama atenção o fato de que os Enfermeiros 3, 4 e 5 responderam que $100 \%$ dos itens estavam adequados.

Tabela 4- Concordância aos pares dos Enfermeiros, através do coeficiente de concordância de Kappa de Cohen. Aracaju, SE, Brasil, 2018.

$\begin{array}{lllll}\text { Enfermeiro } 1 & \text { Enfermeiro 2 } & \text { Enfermeiro } 3 & \text { Enfermeiro 4 } & \text { Enfermeiro } 5\end{array}$

\section{Enfermeiro 1}

Enfermeiro $2 \quad 0.04(0.541)$

Enfermeiro $3 \quad 0.00(1.000) \quad \mathbf{1 . 0 0}(\mathbf{0 . 0 0 0})$

Enfermeiro $4 \quad 0.05(0.244) \quad \mathbf{1 . 0 0}(\mathbf{0 . 0 0 0}) \quad \mathbf{1 . 0 0}(\mathbf{0 . 0 0 0})$

Enfermeiro $5 \quad 0.00(1.000) \quad \mathbf{1 . 0 0}(\mathbf{0 . 0 0 0}) \quad \mathbf{1 . 0 0}(\mathbf{0 . 0 0 0}) \quad \mathbf{1 . 0 0}(\mathbf{0 . 0 0 0})$

Fonte: Os autores

No que se refere aos Dados de Identificação, foi sugerido retirar dados de identificação; inserir "medicações em uso", "comorbidades", “doenças associadas", "doenças pré-existentes", "dia de internação" e "número do prontuário" ou "registro de internação".

$\mathrm{Na}$ dimensão Sinais Vitais recomendaram a inserção dos dados "prostração", “convulsões", “avaliação neurológica", "identificação do olho com alteração pupilar". Foi sugerido também, inserir campo com os parâmetros dos sinais vitais, e na avaliação do nível de consciência, acrescentar as opções "sonolento, "comatoso", "torporoso".

$\mathrm{Na}$ dimensão Necessidade de Oxigenação, sugeriram alterações gráficas, 
identificar o lado da deformidade torácica, inserir parâmetros dos equipamentos utilizados e acrescentar os termos "precórdio calmo", "precórdio dinâmico", “à inspeção do tórax", "CPAP", "BIPAP", "cânula nasal de alto fluxo", "nebulização" e "puff”, sendo estes dois últimos acompanhados de campo para substância utilizada.

Ao serem avaliados os itens relativos à Necessidade de Circulação, foi questionada a alocação do termo "gânglios palpáveis" nessa dimensão e recomendada a adição de campos para hemocomponente e um bloco reservado para avaliação neurológica (Derivação Ventricular Externa).

A respeito da dimensão Necessidade Cutâneo Mucosa, julgou-se a necessidade de reformulação, com sugestões de: inclusão da escala de Braden; "presença e avaliação de curativos", "hidratação da mucosa oral", "língua e dentição"; "caracterização a respeito de incisão cirúrgica e curativo".

Quanto à dimensão Necessidade de Termorregulaçãoos considerou-se a necessidade de inclusão dos termos "pele marmórea", "manta térmica" "termômetro transesofágico" e "hipotermia terapêutica".

Já na dimensão Necessidade de Nutrição e Hidratação, identificou-se a necessidade de retirar o item "balanço hídrico" (já consta em impresso avulso) e definir o período do registro; focar nas vazões das infusões e retirar o quadro relativo à "soroterapia". Incluir o termo "dieta enteral", “dieta parenteral", "gastrostomia", “episódios de êmese". Também foi questionada a forma de aferição do peso e altura das crianças restritas ao leito.

A dimensão Necessidade de Eliminação teve sugestões de acréscimo da "coloração das fezes", "cistostomia" e "bolsa de colostomia". Foi questionado a respeito do período em que o volume urinário e de drenagem será avaliado, e indicada a necessidade de inserir o tempo em que será avaliado o volume das eliminações.

Para a dimensão Necessidade de Higiene foi recomendado inserir dados sobre cabelos, fâneros, hiperemia perineal, glande, prepúcio, presença de parasitos e os termos "sialorreia" e "sangramento" em higiene oral, e retirar o termo "vestes sujas", além de descrever as siglas "PEG", "REG", "BEG".

$\mathrm{Na}$ dimensão Necessidade de Locomoção, houve considerações sobre retirar dados dessa dimensão, pois já estão presentes na escala de Braden de forma avulsa; acrescentar os dados "mudança de decúbito e frequência" dessa mudança em crianças acamadas.

Quanto à Necessidade de Sono $e$ Repouso, os avaliadores julgaram a necessidade de inserir os dados "medicação sedativa" ou "auxiliares do sono".

$\mathrm{Na}$ dimensão Necessidade de Segurança, foi indicado colocar a escala de Morse na íntegra, de preferência a adaptada para crianças; esclarecer o que se refere ao 
cuidador ou à criança; adicionar os termos "acompanhado de" e "necessidade de acompanhamento psicológico".

Foram sugeridas as seguintes alterações para a dimensão Necessidade de Lazer e Recreação: inserir os termos "atividades lúdicas preferidas"; "demonstra interesse pela atividade recreativa"; "não demonstra interesse pela atividade recreativa"; "participa da atividade lúdica"; "não participa da atividade lúdica".

$\mathrm{Na}$ dimensão Necessidades Espirituais, julgou-se necessário o preenchimento dos dados espirituais somente na admissão. Foi recomendado adicionar espaço para registro da religião professada pela família e incluir o termo "necessidade de apoio emocional".

Ajustes de grafia, ortografia, layout, aumento dos espaços dos campos para registros foram sugestões comuns a todas as dimensões avaliadas.

Dessa forma, $57 \%$ dos itens obtiveram o IVC $\geq 70 \%$ sendo considerados validados. Somado a isso, os outros cálculos apresentaram índice de significância maior que o estabelecido, demonstrando não concordância entre os validadores. Devido à utilização da técnica de Delphi no estudo, se fez necessária à realização de mais uma rodada até que todos os itens fossem validados.

Para isso, o instrumento foi alterado com todas as sugestões aceitas, em seguida, foi reformulado o roteiro de avaliação do instrumento, nesse foi explicitado o que ocorreu durante a primeira etapa do processo de validação e sinalizado, na cor vermelha, os itens que ainda não haviam sido validados.

Durante a segunda rodada de avaliação do instrumento não houve perda de nenhum dos experts. Os itens que obtiveram IVC igual a um não foram reavaliados nesta segunda rodada. Dessa forma, o índice de concordância conjunta dos Experts, avaliado através do coeficiente de concordância de Kendall, foi calculado para o total dos itens respondidos na segunda rodada. $\mathrm{O}$ índice de concordância foi igual a 0,96, com p-valor igual a 0,0011, mostrando que a concordância conjunta foi significativa.

$\mathrm{Na}$ Tabela 5 foram avaliados todos os pares de Experts em relação a sua concordância. $\quad$ Os $\quad$ Expert $1, \quad 3 \quad$ e 4 consideraram todos os itens aprovados, onde a concordância entre eles foi perfeita. Apenas o Expert 2 indicou a necessidade de reformulação em alguns itens, porém, no geral ele ainda apresentou concordância significativa com os demais. 
Tabela 5 - Concordância aos pares dos Experts, através do coeficiente de concordância de Kappa de Cohen. Aracaju, SE, Brasil, 2018.

$\begin{array}{llll}\text { Expert } 1 & \text { Expert 2 } & \text { Expert 3 } & \text { Expert } 4\end{array}$

Expert 1

Expert $2 \quad 0.39(0.047)$

$\begin{array}{lll}\text { Expert } 3 & 1.00(<0.01) & 0.39(0.047)\end{array}$

$\begin{array}{llll}\text { Expert } 4 & 1.00(<0.01) & 0.39(0.047) & 1.00(<0.01)\end{array}$

Fonte: Os autores

\section{DISCUSSÃO}

Para a implementação do processo de enfermagem é essencial à organização do serviço. Nesse sentido, durante o período de coleta de dados foi observada a existência de muitos impressos específicos, como: impresso sobre dispositivos em uso, escalas etc. Estes não ficavam anexados ao prontuário do paciente, mas em uma pasta avulsa junto às avaliações de outros pacientes internados. Sendo o prontuário um conjunto de informações de identificação pessoal, familiar, socioeconômica e de saúde do paciente, todos os dados devem estar contidos nele a fim de colaborar para a continuidade do cuidado e facilitar o processo de trabalho. ${ }^{17}$

Assim, visando à revisão e reorganização dos impressos, bem como atender as sugestões dos avaliadores, foram incluídas as escalas de Braden-Q e de Humpty dumpty, além de um quadro de dispositivos em uso, a fim de que as informações sejam preenchidas e arquivadas juntas.
Nesse contexto, o campo "número do prontuário" foi adicionado, a fim de favorecer ao arquivamento correto dos prontuários.

A sugestão de retirada dos dados de identificação não foi atendida, uma vez que a justificativa para tal vai de encontro à cultura de segurança do paciente. A identificação do paciente constitui-se como meta internacional para preservar a segurança do mesmo. O gerenciamento de riscos é considerado um princípio básico para redução de eventos adversos, contrariando a tendência de que o cuidado a pacientes de longa permanência seja discretamente negligenciado pelos profissionais. ${ }^{18}$

Entende-se que os pacientes em unidades de terapia intensiva permanecem em longo regime de internamento. No entanto, os registros devem ser completos e fidedignos diariamente, a fim de atender princípios de legalidade e segurança no sentido de não infringir as obrigações e responsabilidades. 
Grande parte das avaliações se referiram ao uso de dispositivos, e à inclusão de opção dos mesmos no instrumento. A maior parte dos pacientes internados nesse setor, são dependentes de ventilação mecânica ou outras modalidades de oxigenioterapia que interfere na alta. Estudo de revisão mostrou que as terapias com alto fluxo são as mais utilizadas e bem aceitas pelos pacientes, como o CPAP. São principalmente utilizadas nos casos de bronquiolite e insuficiência respiratória e consideradas como um estágio anterior ao uso de ventilação mecânica. ${ }^{19}$

Inicialmente cada dispositivo foi alocado dentro da necessidade correspondente, a fim de adequar ao modelo das NHB. Apesar disso, diante das sugestões foi criado um novo quadro de monitoramento de dispositivos em uso pelo paciente.

A sugestão de introduzir o dado "hemoterapia" foi aceito. Estudos demonstraram que há um crescimento do número de transfusões sanguíneas em hospitais, geralmente indicadas para pacientes graves. Além disso, é importante alertar o profissional de enfermagem sobre a assistência rigorosa e cuidados específicos para pacientes em hemoterapia. ${ }^{20}$

A inclusão do termo "mudança de decúbito não foi aceita, levando em consideração que o mesmo corresponde a uma prescrição de enfermagem, constante na terceira etapa do PE. Como o instrumento em questão trata-se da primeira etapa, sugere-se que as prescrições sejam compiladas em outro instrumento.

Considerando que a maioria dos pacientes internados no setor, possui algum tipo de comprometimento neurológico, a avaliação da função neurológica é imprescindível. Assim, atendendo a solicitações, foi criado um campo para avaliação neurológica, englobando o nível de consciência, a avaliação pupilar e os dispositivos neurológicos em uso, como a DVE.

Um aspecto importante a ser monitorado em pacientes sob cuidados intensivos é o balanço hidrossanguíneo (BH). Realizado nas 24h, constitui-se um instrumento indispensável para avaliação do equilíbrio hídrico do paciente. O manuseio desse instrumento por muitos profissionais, pode provocar erros de cálculo. ${ }^{20}$ Foi sugerido retirar o "quadro de soro" e manter o campo para registro do volume e vazão das infusões. Considerando os riscos quanto ao manuseio do $\mathrm{BH}$, a sugestão foi aceita, sendo recomendado que a planilha de $\mathrm{BH}$ permaneça no prontuário do paciente, e não em pasta avulsa.

Das diversas sugestões de inclusão de termos feitas, os termos "convulsão", "coloração das fezes", "alimentação enteral”, "regime terapêutico", "doenças preexistentes", "atividades recreativas ou de lazer" foram adicionados ao instrumento, uma vez que os demais não constavam na 
Taxonomia NANDA I, linguagem escolhida como embasamento para direcionar a construção de diagnósticos de enfermagem. ${ }^{12}$

No que se refere ao sono e repouso, foi sugerida a adição dos dados "medicação sedativa, ou de auxiliares do sono". Estas sugestões foram incorporadas ao instrumento. Estudos identificaram que "padrão do sono prejudicado" e "insônia" são diagnósticos de enfermagem prevalentes em unidades de terapia intensiva. ${ }^{22}$ Isso demonstra a importância da avaliação desses dados, além de direcionar intervenções para resolução desses diagnósticos.

Todas as considerações gráficas, ortográficas e de layout foram atendidas, incluindo a criação de legendas com descrição das siglas utilizadas.

Durante a segunda rodada somente a expert 2 fez novas sugestões em alguns itens, os demais experts consideraram todos os itens como aprovado após a reformulação do instrumento.

\section{CONCLUSÃO}

As dimensões que sofreram maiores alterações foram: necessidade de oxigenação e necessidade de nutrição e hidratação. Dos 70 itens analisados pelos experts e assistenciais, 40 e 65, respectivamente obtiveram IVC $\geq 70 \%$ na primeira rodada e ambos julgaram os 70 itens na segunda rodada, sendo atribuído um IVC $\geq 70 \%$ para todos eles.
O estudo teve como dificuldade o tempo dispendido para que os experts retornassem suas considerações aos pesquisadores tanto na primeira quanto na segunda rodada. $\mathrm{O}$ uso dos meios virtuais de comunicação para realização da pesquisa foi um fator positivo, vez que tornou a pesquisa de baixo custo, ao passo que conseguiu ter a avaliação de experts de vários estados brasileiros.

Dessa forma, foi possível validar um instrumento de coleta de dados de enfermagem pautado em um referencial teórico a fim de direcionar e facilitar a assistência de enfermagem, permitindo que o uso do mesmo direcione para construção de diagnósticos de enfermagem e desenvolvimento das fases posteriores do processo de enfermagem.

\section{REFERÊNCIAS}

1. Guedes DMB, Rossato LM, Oliveira EA. Diagnósticos de enfermagem mais frequentes em uma unidade de terapia Intensiva pediátrica.. Rev Enferm UFSM. [Internet]. 2015 [acesso em 12 mar 2019]. Jul-Set; 5(3):476-485. Disponível em: https://periodicos.ufsm.br/reufsm/artic le/view/16471/0 DOI: $10.5902 / 2179769216471$

2. Estatuto da Criança e do Adolescente, LEI No 8.069 de 13 de Julho de 1990. 
Dispõe sobre o Estatuto da Criança e do Adolescente e dá outras providências. [Internet].; 1990 [acesso em 09 mar 2019]. Available from: www.planalto.gov.br. Disponível em: http://www.planalto.gov.br/ccivil_03/1 eis/18069.htm

3. Marques DKA, Silva KL, Nobrega MML. Escolares hospitalizados: proposta de um instrumento para coleta de dados à luz da teoria de Horta. Rev Gaúcha Enferm. [Internet]. 2016 [acesso em 27 fev 2019]; 37(esp):e2016-0038. Disponível em: http://www.scielo.br/pdf/rgenf/v37nsp e/0102-6933-rgenf-1983-

$\underline{14472016 \operatorname{esp} 2016-0038 . p d f}$ DOI: 10.1590/1983-1447

4. Ramalho Neto JM, Fontes WD, Nobrega MML. Instrumento de coleta de dados de enfermagem em Unidade de Terapia Intensiva Geral. Rev Bras Enferm. [Internet]. 2013 [acesso em 27 fev 2019]; 66(4):535-542. Disponível em: http://www.scielo.br/pdf/reben/v66n4/ v66n4a11.pdf DOI: 10.1590/S003471672013000400011

5. Soares MI, Resck ZMR, Terra FS, Camelo SHH. Sistematização da assistência de enfermagem: facilidades e desafios do enfermeiro na gerência da assistência. Esc. Anna Nery Rev. Enferm. [Internet]. 2015 [acesso em
15 mar 2019] Jan- Mar; 19(1): 47-53. http://www.scielo.br/pdf/ean/v19n1/14 14-8145-ean-19-01-0047.pdf DOI: 10.5935/1414-8145.20150007

6. Perão OF, Zandonadi GC, Rodríguez $\mathrm{AH}$, Fontes MS, Nascimento ERP, Santos EKA. Segurança do paciente em unidade de terapia intensiva de acordo com a teoria de Wanda Horta. Cogitare Enferm. [Internet]. 2017 [acesso em 05 fev 2019]; 22(3): e45657, 2017. Disponível em: https://revistas.ufpr.br/cogitare/article/ view/45657

DOI: $10.5380 /$ ce.v22i3.45657

7. Melo LL, Santos MS, Duran ECM. Unidade de terapia intensiva pediátrica: diagnósticos e intervenções de enfermagem mais frequentes. Rev enferm UFPE on line. [Internet]. 2014 [acesso em 05 fev 2019]; 8(supl 1):2342-2349. Disponível em: https://periodicos.ufpe.br/revistas/revi staenfermagem/article/view/9924/102 10 DOI: $10.5205 / \mathrm{reuol} .5927-50900-$ 1-SM.0807supl201420

8. Queirós PJP. Cuidar: da condição de existência humana ao cuidar integral profissionalizado. Revista de Enfermagem Referência. [Internet]. 2015 [acesso em 14 mar 2019]; Serie IV(5):139-146. Disponível em: https://www.researchgate.net/publicati $\underline{\text { on/279449765_Cuidar_da_condicao_d }}$ 
e_existencia_humana_ao_cuidar_integ $\underline{\text { ral_profissionalizado }}$

DOI:

10.12707/RIV14079

9. Conselho Federal De Enfermagem (COFEN). Resolução COFEN $\mathrm{n}^{\circ}$ 358/2009, de 15 de outubro de 2009. Dispõe sobre a Sistematização da Assistência de Enfermagem e a implementação do Processo de Enfermagem em ambientes, públicos ou privados, em que ocorre o cuidado proffisonal de enfermagem, e dá outras providências. [Internet]. 2009 [acesso em 14 mar 2019]. Disponível em: www.cofen.gov.br/resoluo-cofen3582009 4384.html.

10. Schmitz EL, Gelbcke FL, Bruggmann MS, luz SCL. Filosofia e marco conceitual: estruturando coletivamente a sistematização da assistência de enfermagem. Rev Gaúcha Enferm. [Internet]. 2016 [acesso em 14 mar 2019]; 37(esp): e68435. Disponível em:

https://www.scielo.br/pdf/rgenf/v37ns pe/0102-6933-rgenf-198314472016esp68435.pdf DOI: 10.1590/1983-1447.2016.esp.68435.

11. Horta WA. Processo de Enfermagem. Rio de Janeiro: Guanabara Koogan; 2011.

12. Internacional N. Diagnósticos de enfermagem da NANDA: definições e classificação 2015-2017. Porto Alegre: Artmed; 2015.

13. Scarparo AF, Laus AM, Azevedo ALCS, Freitas MRI, Gabriel CS, Chaves LDP. Reflexões sobre o uso da Técnica de Delphi em pesquisas na enfermagem. Rev Rene. [Internet]. 2012 [acesso em 10 mar 2019]; 13(1):242-251. Disponível em: http://www.periodicos.ufc.br/rene/arti cle/view/3803

14. Silva Júnior SD, Costa FJ. Mensuração e Escalas de Verificação: uma Análise Comparativa das Escalas de Likert e Phrase Completion. PMKT- Revista Brasileira De Pesquisas de Marketing, Opinião e Mídi. [Internet]. 2014 [acesso em 10 mar 2019]; 15. Disponível em: http://sistema.semead.com.br/17semea d/resultado/an_resumo.asp?cod_trabal $\underline{\mathrm{ho}=1012}$

15. Kendall MG. Rank correlation methods London: Griffin; 1948.

16. Cohen JA. Coefficient of agreement for nominal scales. Educational and Psychological Measurement. [Internet]. 1960 [acesso em $10 \mathrm{mar}$ 2019]; $\quad 20(1) . \quad$ DOI: 10.1177/001316446002000104

17. Conselho Federal de Medicina, RESOLUÇÃO n ${ }^{\circ} 1.638 / 2002$ de 09 de Agosto de 2002. Define prontuário médico e torna obrigatória a criação da 
Comissão de Revisão de Prontuários http://www.revistaeletronicafunvic.org nas instituições de saúde. [Internet]. 2002 [acesso em 18 fev 2019]. /index.php/c14ffd10/article/view/83/7

Disponível

em:

http://www.portalmedico.org.br/resolu coes/cfm/2002/1638_2002.htm.

18. Gomes APTS, Querido DL, Silva GRGd, Almeida LFd, Rocha RG. Identificação do paciente em neonatologia para assistência segura. Cogitare Enferm. [Internet]. 2017 [acesso em 12 fev 2019]; 22(3): e49501. Disponível em: https://revistas.ufpr.br/cogitare/article/ view/49501

DOI: $10.5380 /$ ce.v22i3.49501

19. Rosa NC, Rolim R, Almeida TRAd, Carr AMG. Uso da oxigenoterapia de alto fluxo em pediatria e neonatal: revisão de literatura. Revista Saúde. [Internet]. 2017 [acesso em $12 \mathrm{fev}$ 2019]; 11(1-2):91-102. Disponível em:

http://revistas.ung.br/index.php/saude/ article/view/2342/2209

20. Silva PAR, Assis DCM, Silva CR. Conhecimento de profissionais de enfermagem sobre atuação em hemotransfusão. Rev Ciên Saúde. [Internet]. 2017 [acesso em $12 \mathrm{fev}$ 2019]; 2(2):15-24. Disponível em: $\underline{0}$

21. Melo EM, Sales ICF, Almeida DT, Lima FET, Veras JEGLF, Studart RMB. Avaliação dos registros de enfermagem no balanço hídrico de pacientes em unidade de terapia intensiva. Rev Enferm UFPI. [Internet]. 2014 [acesso em $18 \mathrm{fev}$ 2019] Out-Dez; 3(4):35-41. Disponível em: https://pesquisa.bvsalud.org/portal/res ource/pt/bde-30795 DOI: 10.1590/S1414-81452012000300021

22. Ferreira AM, Rocha EN, Lopes CT, Bachion MM, Lopes JdL, Barros ALBLd. Diagnósticos de enfermagem em terapia intensiva: mapeamento cruzado e Taxonomia da NANDA-I. Rev Bras Enferm. [Internet]. 2016 [acesso em 19 fev 2019] Mar-Abr; 69(2):307-315. Disponível em: https://www.scielo.br/pdf/reben/v69n2 10034-7167-reben-69-02-0307.pdf DOI: $10.1590 / 0034-$ 7167.2016690214i Submissão: 2020-12-16 Aprovado: 2021-05-06

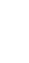

